\title{
Current status and perspectives in lung transplantation
}

\author{
P. Vitulo
}

Monaldi Arch Chest Dis 2004; 61: 4, 197-198.

ISMETT/UPMC (Istituto Mediterraneo per i Trapianti e Terapie ad Alta Specializzazione/University of Pittsburg Medical Center) Palermo - Italy.

Correspondence: Dr. Patrizio Vitulo MD; Pulmonology and Lung Transplant Unit; ISMETT/UPMC (Istituto Mediterraneo per $i$ Trapianti e Terapie ad Alta Specializzazione/University of Pittsburg Medical Center); Via Tricomi, 1 - 90127 Palermo; e-mail: pvitulo@ismett.edu

More than two decades after the very first lung transplant was successfully performed by the Toronto Lung Transplant Group, lung transplantation (LTX) has become a valid approach to treatment of end-stage parenchymal and vascular lung disease due to the satisfactory results achieved in terms of survival and quality of life.

According to the Registry of the International Society of Heart and Lung Transplantation, the current 1-, 2- and 5-yr overall actuarial survival following LTX is $77.1 \%, 61.1 \%$ and $47.5 \%$ respectively [1]. Long term survival is mainly affected by the onset of obliterative bronchiolitis, an irreversible form of chronic lung rejection. Although the survival rates following LTX are considerably lower than after liver or heart transplantation, LTX still provides a remarkable advantage in terms of quality of life. These encouraging results have widened the indications for LTX, thus resulting in an increased number of patients awaiting an operation.

The indications for LTX seem to have a different distribution according to which country they are in. In the US, for example, Chronic Obstructive Pulmonary Disease accounts for $48 \%$ of all the patients listed for LTX, Idiopathic Pulmonary Fibrosis for 17\% and Cystic Fibrosis for 16\%; in Italy, in contrast, the same indications account for $28 \%$, $23 \%$ and $21 \%$ of the patients referred for LTX, respectively. This is in part due to different organ allocation policies. In the US, the allocation of lungs is based on the time accrued by each patient on the waiting list; in the majority of European Countries, however, the selection of the recipient is made by the local transplant team. The latter system allows for a disease-oriented selection, and takes into account the severity of each patient's prognosis and clinical status.

The number of patients on the waiting list seems to increase at a higher rate in the US than in Europe [2]. This could reflect a more careful patient selection in the European centres. Nevertheless, this data needs to be carefully reviewed, since a single candidate could be registered in more than one centre in the US, while this rarely occurs in Europe.
Despite these differences, the majority of lung transplant centres are facing the condition of a substantially stable number of organs available for LTX versus a growing trend of patients listed for transplant. The imbalance between demand and offer is reflected by the worrying increase in the number of patients dying on the waiting list.

Organ shortage implies several ethical, economic and logistic issues and requires the optimisation of the process of recipient selection, organ procurement strategy, and graft preservation, in order to allow a safer use of the so-called "marginal donors".

The selection process of LTX candidates should take in account the following aspects: stage, natural history and recent evolution of the underlying disease; general functional status of the patient (nutrition, walking capacity); an estimate of the waiting time based on the type of transplant, the $\mathrm{ABO}$ and dimensional matching, and the average waiting time in the selected centre.

Despite the fact that recipient selection plays a key role in defining the outcome of LTX, there is surprisingly little evidence on this topic. The lack of prognostic factors for each single disease irremediably affects the process of a proper timing for referral to LTX, which is essential for a correct and equitable organ allocation.

In the case of pulmonary vascular disease, the prognosis of patients with associated congenital heart disease is generally better than those with idiopathic pulmonary hypertension, despite the fact that in both cases hemodynamic and respiratory parameters are heavily altered.

The paper from G. Callegari and co-workers published in this issue of Monaldi Archives [3] reports the experience of the Pavia Lung Transplant Centre with a large group of patients affected by Eisenmenger Syndrome who were listed for LTX. The authors retrospectively investigated for predictors of survival among clinical, respiratory, and hemodynamic parameters. No significant differences were noted between the group of survivors and those who died. An unfavourable trend was seen in those patients who had heart defects and 
previous cardiac surgery. The authors conclude that it is very difficult to predict the outcome of patients with this kind of pulmonary disease.

New appealing prognostic predictors in patients with Eisenmenger Syndrome are the increased blood concentration on brain natriuretic peptide and the absence of a response to vasoreactive test during right heart catheterisation. The latter is described in a recent paper by MC Post [4]: in a series of 21 consecutive patients affected by Eisenmenger syndrome, a significant vasoreactivity after inhalation of NO during right heart catheterization correlated with a significantly better mid-term prognosis.

In the future, the progressing knowledge of the mechanisms of local cell proliferation signals and the development of new immunosuppressive drugs will positively affect the prevention and the treatment of chronic lung rejection.

Other interesting and promising areas of research are the advances in lung preservation, living-related LTX, and the utilization of non-beating-heart donors.

Since xenotransplantation does not appear to be an achievable goal in the next few decades due to strong immunological barriers between species, the induction of tolerance to donor-specific antigens seems the more promising road to rejectionfree LTX. The administration of depleting or blocking agents and immunomodulatory cell populations could prolong graft function and minimize drug toxicity.

\section{References}

1. Trulock EP, Edwards LB, Taylor DO, Boucek MM, Keck BM, Hertz MI. The Registry of the International Society for Heart and Lung Transplantation: twentyfirst official adult heart transplant report - 2004. $J$ Heart Lung Transplant 2004; 23: 804-15.

2. The Organ Procurement and Transplantation Network. UNOS Registry Data. www.unos.org. Accessed June 2003.

3. Callegari G, D'Armini AM, Baiardi P, Viganò M, Fracchia C. Predictors of mortality in patients with Eisenmenger syndrome and admission to lung transplantation waiting list. Monaldi Arch Chest Dis 2004; 4: 199-202.

4. Post MC, Janssens S, Van de Werf F, Budts W. Responsiveness to inhaled nitric oxide is a predictor for mid-term survival in adult patients with congenital heart defects and pulmonary arterial hypertension. Eur Heart J 2004 Sep; 25 (18): 1651-6.

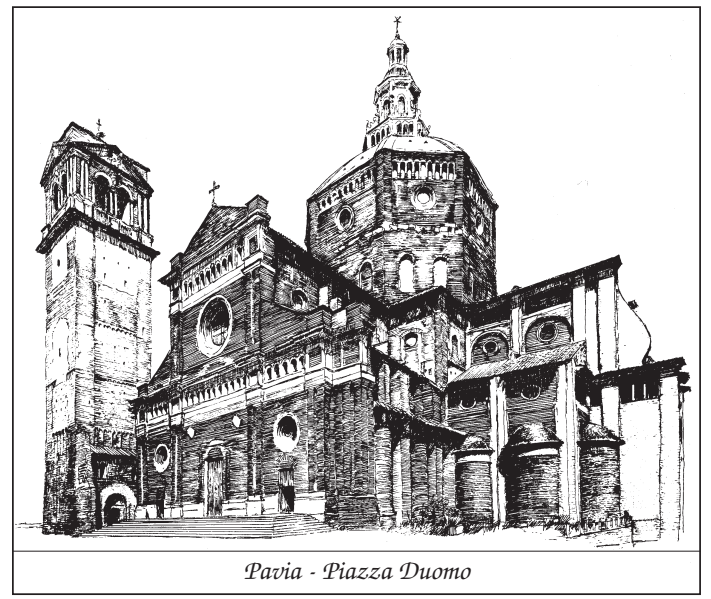

